\section{Psoriasis: dreifacher Erfolg für IL-17-Antikörper}

\author{
Der neu zugelassene IL-17A-Antikörper Ixekizumab ist in drei Phase-III-Studien \\ mit Psoriasispatienten untersucht worden.
}

m April hat mit Ixekizumab ein zweiter Antikörper gegen IL-17A die Zulassung zur Behandlung der mittelschweren bis schweren Psoriasis erhalten. Die Wirksamkeit des humanisierten monoklonalen Antikörpers wurde unter anderem in den Phase-III-Studien UNCOVER-1 bis -3 mit zusammen mehr als 3.700 Patienten belegt. In UNCOVER-2 und -3 war Ixekizumab nach zwölf Wochen sowohl einer Placebo- als auch einer Etanerceptbehandlung überlegen [Griffiths $\mathrm{CE}$ et al. Lancet. 2015;386:541-51]. Die Ergebnisse der drei Studien nach bis zu 60-wöchiger Dauer wurden nun publiziert.

In UNCOVER-1 (1.296 Patienten, mittlerer PASI zu Beginn ungefähr 20) hatte die zwölfwöchige Induktionsphase mit Ixekizumab, wie auch in den beiden anderen Studien, eine signifikante Besserung der Psoriasis bewirkt. Mit einer Dosis von $80 \mathrm{mg}$ alle zwei oder alle vier Wo- chen erreichten $81,8 \%$ respektive $76,4 \%$ der Patienten im „static Physician's Global Assessment" (sPGA) einen Wert von 0 oder 1 (keine oder minimale Psoriasis) und $89,1 \%$ und $82,6 \%$ eine mindestens $75 \%$ ige Besserung im Psoriasis Area and Severity Index (PASI 75) (mit Placebo entsprechende Raten: 3,2\% und 3,9\%).

Teilnehmer von UNCOVER-1 und -2, deren sPGA nach zwölf Wochen 0 oder 1 betrug $(n=1.126)$, wurden $z u$ diesem Zeitpunkt erneut randomisiert und mit Ixekizumab $80 \mathrm{mg}$ alle vier oder alle zwölf Wochen oder mit Placebo behandelt. Nach insgesamt 60 Wochen hatten $73,8 \%, 39,0 \%$ und 7,0\% der Patienten weiterhin einen sPGA von 0 oder 1 . Bei vierwöchentlicher Antikörpergabe erlebte keiner der Patienten eine Exazerbation mit einem sPGA von 3 oder mehr.

In der Verlängerungsphase von UNCOVER-3 wurden 771 Patienten aus den beiden Antikörpergruppen mit Ixekizumab alle vier Wochen weiterbehandelt. In Woche 60 hatten mindestens $73 \%$ einen sPGA-Score von 0 oder 1 und mindestens $80 \%$ einen PASI 75.

$\mathrm{Zu}$ den unerwünschte Wirkungen unter der Antikörpertherapie zählten Neutropenien (darunter acht Grad 3 und zwei Grad 4), orale Candidainfektionen und chronische entzündliche Darmerkrankungen (CED, 11 vs. 3 unter Placebo) sowie zwei vaskuläre Todesfälle.

Fazit: Die Neutralisierung von IL-17A durch Ixekizumab hat sich in drei Phase-3-Studien als wirksame Therapie der mittelschweren bis schweren Psoriasis erwiesen. Durch eine vierwöchentliche Behandlung konnten die hohen Ansprechraten über den gesamten Studienzeitraum von 60 Wochen erhalten werden. Als Nebenwirkungen können Neutropenien, Candidainfektionen und CED auftreten. Dr. Beate Schumacher

Gordon KB et al. Phase 3 Trials of Ixekizumab in Moderate to Severe Plaque Psoriasis. N Engl J Med. 2016;375:345-56.

\title{
Acanthosis nigricans und Hyperandrogenismus
}

Eine 27-jährige Frau stellte sich wegen seit zwei Jahren bekannter Acanthosis nigricans (Abb. A, B), Hirsutismus und Amenorrhö vor. Der Insulinspiegel lag bei über $200 \mu \mathrm{U} / \mathrm{ml}$ (Norm: 2-13 $\mu \mathrm{U} /$ $\mathrm{ml}$ ), das Plasma-Testosteron war mit $649 \mathrm{ng} / \mathrm{ml}$ massiv erhöht, es fanden sich Antikörper gegen Thyreoperoxidase (TPO) sowie antinukleäre Antikörper (ANA) mit einem Titer von 1:64. Die Werte für Blutzucker und $\mathrm{HbA1c}$ waren normal, eine Malignomsuche blieb negativ.

Bei der Wiedervorstellung nach sechs Monaten hatte die Patientin deutlich an Gewicht verloren. Nüchtern ergab sich ein Blutzuckerwert von 174 mg/dl, der HbA1c-Wert lag bei 11,2\%. Der C-Peptid-Spiegel war mit 7,3 ng/ ml leicht erhöht, der Test auf Insulin-Antikörper negativ. Man begann eine Therapie mit Metformin, unter der sich sechs Monate später Hypoglykämien einstellten. Der Titer der Insulinrezeptor-Antikörper war mit 1:1.280 erhöht. Unter der Diagnose eines Insulinresistenzsyndroms vom Typ B begann man eine Therapie mit Prednisolon und Azathioprin, worunter sich binnen sechs Wochen Blutzucker, Testosteron und Insulinspiegel normalisierten und die Hautbefunde verbesserten (Abb. C, D).

Eine Acanthosis nigricans kann durch endokrine, metabolische, genetische und paraneoplastische Faktoren hervorgerufen werden. Das Insulinresistenzsyndrom Typ B ist eine seltene Autoimmunerkrankung, bei der Antikörper gegen den Insulinrezeptor zirkulieren, die zu Störungen des Blutzuckerstoffwechsels, extre- mer Insulinresistenz, Acanthosis nigricans und Hyperandrogenismus führen. Hier ist der Spezialist gefragt. Prof.Dr. H. S. FüeßI

Lauria MW, Saad MJ. Acanthosis nigricans and insulin resistance. N Engl J Med. 2016;374:e31
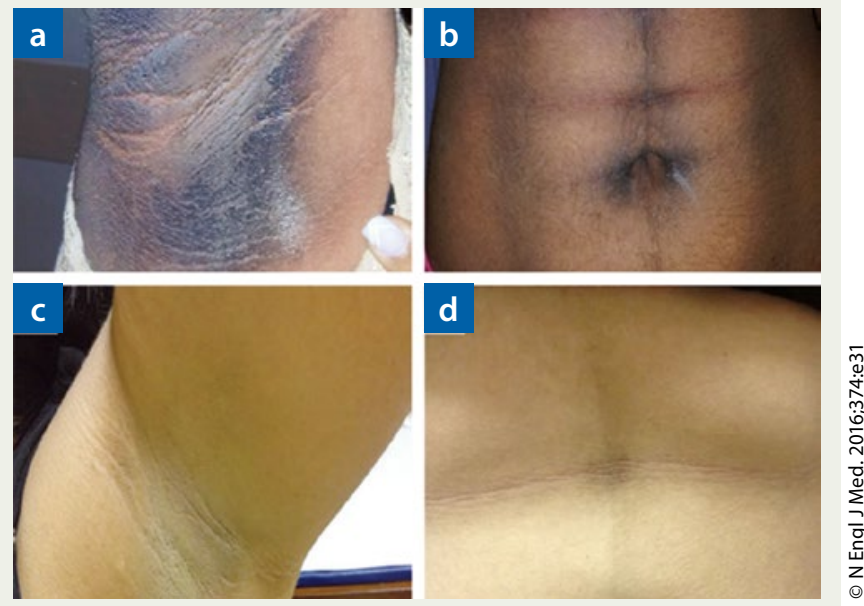

A, B: Acanthosis nigricans bei einer 27-jährigen Frau; C, D: Zustand nach sechs Wochen Arzneimitteltherapie 